\title{
Develop a validated anesthesia quality assessment to evaluate patients postoperatively
}

\begin{abstract}
Objective: The study is aimed to develop a well-validated anesthesia quality assessment to evaluate patients postoperatively.

Materials and Methods: After securing Institutional Review Board approval, the study was registered via ClinicalTrials.gov (NCT 0307996). The Siriraj Anesthesia Quality Assessment Questionnaire was developed and validated under the conceptual/theoretical framework of Maslow's hierarchy of needs, Hospital Consumer Assessment of Healthcare Providers and System, Patient Satisfaction Questionnaire, Service Quality Questionnaire and Quality of Recover after Anesthesia. A total of 476 qualified responses with obtained consent were included in the study to verify the appropriateness of the tool.
\end{abstract}

Results: The study involved 397 qualified patients (83.4\%), aged 47.6 \pm 16.6 , representing all anesthesia services. Significantly, a binary logistic regression revealed the predicted dependent variables as Process of Care (4.39 \pm 0.51$)$ and Physical Support $(4.35 \pm 0.66)$ in comparison with a sequence of Accessibility and Convenience (4.33 \pm 0.56$)$, Communication

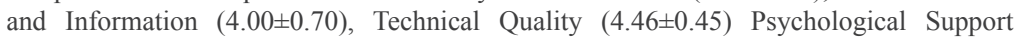
$(4.57 \pm 0.65)$ and Loyalty $(4.52 \pm 0.68)$ in anesthesia quality assessment.

Conclusion: Process of Care and Physical Support appeared to be significantly valued features, whereas Communication and Information, Accessibility and Convenience, Technical Quality and Psychological Support were found to be weak points in anesthesia quality assessment.
Volume I2 Issue I - 2020

\section{Sudta Parakkamodom,' Phongthara Vichitvejpaisal,' Papiroon Noitasaeng,' Suthipol Udompunturak, ${ }^{2}$ Somchai Amornyotin'}

'Department of Anesthesiology, Faculty of Medicine Siriraj Hospital, Mahidol University, Bangkok, Thailand

${ }^{2}$ Office of Research Promotion, Faculty of Medicine Siriraj Hospital, Mahidol University, Bangkok, Thailand

Correspondence: Somchai Amornyotin, Department of Anesthesiology, Faculty of Medicine Siriraj Hospital, Mahidol University, Bangkok, Thailand, Tel (+66) 24197990, Email somchai.am@mahidol.ac.th

Received: February 20, 2020 | Published: February 26, 2020

\section{Introduction}

Siriraj Hospital is a tertiary, university hospital with more than 2,200 beds. Its Department of Anesthesiology employs 68 boardcertified anesthesiologists, 81 nurse anesthetists and 126 trainees (79 residents, 11 fellows and 36 nurse anesthetist students) responsible for the care of more than 50,000 patients annually. ${ }^{1}$ Since defining and measuring quality of healthcare service is a real challenge for all institutes in these fields, many organizations have tried to work out intensively, as everyone desires an excellent medical response. Anesthesia is likened to a business firm, which needs key performance indicators (KPIs) to measure its operational goals. According to Maslow's hierarchy of needs, human pleasure is classified as physiological, safety, belongings, esteem and self-actualization. ${ }^{2}$ Likewise, patient satisfaction seems to cover all these characteristics. As a result, their contentment becomes a crucial factor for determining the quality of care, particularly in anesthesia procedures. This includes many processes in peri-anesthesia management such as patient safety in operating theatre, pain controlling and mutually convenient interrelationship.

In those days, patients showed utmost respect for doctors, probably seen as divine gods with magical power to alleviate their mental and physical anguish from diseases. In many places, doctorpatient relationship has advanced to a higher stage of culture and become social norms that link them ever since. Therefore, for fear of threatening future care, patients might have been unwilling to criticize their benefactors. By and large, they therefore showed intense good loyalty, consideration and faith in their doctors. Consequently, it was difficult to assess patients' genuine perspectives, particularly their satisfaction on anesthesia care service. In addition, in the old days, many studies using nonvalidated instruments or poorly developed tools claimed to accurately assess satisfaction with anesthesia, which could have led to biased and inaccurate outcomes. ${ }^{3}$ As a result, investigators worked on literature research for outstanding, wellvalidated tools that fit our norms. Presently, there are many leading quality assessment tests in healthcare services; however, only four instruments were chosen for this study.

The Hospital Consumer Assessment of Healthcare Providers and System (HCAHPS), ${ }^{4}$ a 33 -item survey in three dimensions, comprises nurse and doctor communication, medical communication, responsiveness of hospital staff, pain management, discharge information, care transition (17 items); cleanliness and quietness of hospital environment ( 2 items); as well as overall rating of hospital and willingness to recommend hospital (2 items). The Patient Satisfaction Questionnaire (PSQ), ${ }^{5}$ an 18 -item questionnaire in seven dimensions, consists of general satisfaction, technical quality, interpersonal manner, communication, financial aspects, time spent with doctor as well as accessibility and convenience.

The Service Quality Questionnaire (SERVQUAL), ${ }^{6}$ a questionnaire related to hospital services in five dimensions, is composed of tangibles, reliability, responsiveness, assurance and empathy. The Quality of Recover after Anesthesia 40 (QoR-40), ${ }^{7}$ a $40-$ item questionnaire concerning good quality recovery after anesthesia 
and surgery, includes emotional states, physical and physiological support, physical independence and pain management. Investigator has developed a tool called the Siriraj Anesthesia Quality Assessment Questionnaire (Si-2-AQ) and planned to use this well-validated questionnaire routinely in postoperative patients.

The Si-2-AQ, a simply 40-item tool in seven dimensions, comprises Accessibility and Convenience (5 items): politeness, attention, consultation, participation and expectation; Communication and Information (10 items): identification, education-in general preop/ intraop/postoperative, question, continuing care-in general/ recovery room/ward/home; Process of care (8 items): understanding, satisfaction, concern, perioperative safety, preference, respectfulness, postoperative pain service and perioperative security; Technical Quality (9 items): pain, discomfort, sore throat, hoarseness of voice, nausea, vomiting, deglutition disorder, itching and dysuria; Psychological Support (4 items): anxiety, depression, confusion and insomnia; Physical Support (2 items): cleanliness and quiet atmosphere; and Loyalty (2 items): recommendation and level of satisfaction (Table 1).

Table I Details of healthcare quality assessment tests

\begin{tabular}{|c|c|c|c|c|}
\hline HCAHPS & PSQ- 18 & SERVQUAL & QoR40 & Si-2-AQ \\
\hline \multirow[t]{2}{*}{ Communication with Nurses } & & & & Accessibility \& Convenience \\
\hline & Communication & Empathy & Emotional state & \\
\hline Communication with Doctors & & & & Communication \& Information \\
\hline \multirow[t]{2}{*}{ Responsiveness of Hospital Staff } & Interpersonal Manner & & Physical support & Process of Care \\
\hline & & Assurance & & \\
\hline \multirow[t]{2}{*}{ Pain management } & & & Pain & Technical Quality \\
\hline & Technical Quality & & & \\
\hline Communication about Medicines & & Responsiveness & Physical support & Psychological Support \\
\hline \multicolumn{5}{|l|}{ Cleanliness of Hospital environment } \\
\hline Quietness of Hospital Environment & General Satisfaction & Tangible & Physical comfort & Physical Support \\
\hline Discharge Information & Time Spent with Doctor & Responsiveness & & \\
\hline Overall Rating of This Hospital & Financial Aspects & & & \\
\hline \multirow[t]{2}{*}{ Willingness to Recommend This Hospital } & & Reliability & Physical support & Loyalty \\
\hline & Accessibility and Convenience & & & \\
\hline Care transition & & Responsiveness & Physical independence & \\
\hline
\end{tabular}

\section{Materials and methods}

This manuscript adheres to the applicable STROBE guidelines. After securing Siriraj Institutional Review Board (IRB) approval ( $\mathrm{Si}$ 790/2016), investigators registered clinical study via ClinicalTrials. gov (NCT 0307996) on January 25, 2017 prior to the start of the trial and any patient enrollment. Written informed consent was obtained from all subjects, their legal surrogates, the parents or legal guardians for minor subjects, or the requirement for written informed consent was waived by the IRB. This research and development project was performed at the Department of Anesthesiology, Siriraj Hospital, and lasted six months from January to July 2016. The conceptual/ theoretical framework was based on Maslow's hierarchy of needs, HCAHPS, PSQ-18, SERVQUAL and QoR-40. This project had two parts: the first phase: development and validation of the $\mathrm{Si}-2-\mathrm{AQ}$, and the second phase: use of the Si-2-AQ on the target group.

Phase I: Development and validation of the Si-2-AQ

Investigators developed a 40 -item $\mathrm{Si}-2-\mathrm{AQ}$ covering essential aspects of the post-operative recovery process with five responses on Likert scale: $1=$ very poor, $2=$ poor, $3=$ fair, $4=$ good and $5=$ very good. We considered that consumers were satisfied with our services with higher scores $>3.4$ (interval $=0.8$ of 5 Likert scale). The dimension of Loyalty was determined for correlation with the overall satisfaction, with a 95\% Confidence Interval of completely responsive scores in six measurements as lower (90.0\%) and upper (94.8\%).
A prototype was approved for its operationization, namely, face and content validity (Index of Item-Objective Congruence, IOC) by three professors with at least 30 years of experience in clinical anesthesia practice (this lasted two weeks). The first try-out for objectivity (comprehensibility and readability) was performed in five nurse anesthetists with at least 10 years of experience (this also lasted two weeks). Feedback from experts' open-ended questionnaires was explored and contents analyzed. Afterwards, the second try-out for internal consistency (Cronbach's reliability) was accomplished faceto-face amongst 30 volunteered patients undergoing elective surgery under general or regional anesthesia (this part lasted four weeks). The appropriateness of the tool was tested and re-tested until an IOC $>0.9$ and reliability $>0.8$ were established.

Phase II: use of the Si-2-AQ on the target group

Preoperatively, anesthesia providers made routine visits to patients at wards for discussion of anesthesia preferences, physical and mental assessment, and premedication. On the day after the surgery, a nurse anesthetist called on each patient to explain details of the study and obtain the informed consent. This was to ensure that they could, as much as possible, separate between the outcomes of anesthesia from surgical service. Afterwards, each participant responded to a questionnaire privately (this lasted 15 minutes). Co-investigators then collected and saved data in a computer, and a statistician who was not involved in the project analyzed the records. 
In-patient volunteers, fully conscious, literate, and communicable, ASA I-III, aged 18-65, without severe diseases such as congestive heart failure or end-stage renal disease undergoing elective surgery and requiring anesthesia care, were allocated in the study. Whether they participated did not affect their postoperative care in any way. The exclusion criteria were patients showing unwillingness or refusing to criticize their caregivers. The withdrawal criteria were those who desired to leave the study at any time. Out of 50,000 patients, we excluded $20 \%$ outpatients and $8 \%$ patients under 18 and over 65 . Therefore, the in-patient sampling was $72 \%$ of $50,000=35,891$. The sample size calculated by Taro Yamane (1967) with a proportion of 0.05 at the $95 \%$ Confidence Interval was as follows:

$$
\begin{gathered}
n=\frac{N}{1+N(e)^{2}} \\
n=\frac{35891}{1+35891(0.05)^{2}}
\end{gathered}
$$

Where $\mathrm{n}=$ the sample size

$$
\begin{aligned}
\mathrm{N} & =\text { the population } \\
\mathrm{e} & =\text { the allowable sampling error } \\
& =396
\end{aligned}
$$

We added $20 \%$ for missing data $=476$

Then the total of 476 patients or $1.3 \%$ of population were randomized and included in the project.

\section{Statistical analysis}

Demographic data were expressed as percentage, mean and standard deviation. For objectivity of the test, nurse anesthetists' opinions were collected and analyzed for its comprehensibility. A Binary Logistic Regression was used to verify the factors related to patients' perspectives. A $\mathrm{p}<0.05$ is considered to be statistically significant difference.

\section{Results}

A total of 476 qualified responses were 397 (83.4\%) complete and $79(16.6 \%)$ incomplete data. Demographic characteristics were as follows: 103 (25.9\%) males and 294 (74.1\%) females; aged 47.6 16.6 where $<65$ years were accounted for by $287(72.3 \%)$ and $\geq 65$ years, $110(27.7 \%)$; service in obstetrics $117(29.5 \%)$, gynecology 104 (26.2\%), head \& neck $68(17.1 \%)$, urology $57(14.4 \%)$, orthopedic 35 $(8.8 \%)$ and others $16(4.0 \%)$; under anesthesia in general $232(58.2 \%)$, regional $139(35.0 \%)$ and combined $26(6.6 \%)$; with duration of $1.4 \pm 0.5$ hours where $<2$ hours were accounted for by $274(69.0 \%), \geq 2$

\begin{tabular}{|c|c|c|c|}
\hline & & Responses (\%) & mean $\pm S D$ \\
\hline \multicolumn{4}{|l|}{ Gender } \\
\hline & male & $103(25.9)$ & \\
\hline & female & $294(74.1)$ & \\
\hline \multirow[t]{3}{*}{ Age $(y r)$} & & & $47.6 \pm 16.6$ \\
\hline & $|5-6|$ & $287(72.3)$ & \\
\hline & $>61$ & $110(27.7)$ & \\
\hline \multicolumn{4}{|l|}{ Service unit } \\
\hline & obstetrics & II 7 (29.5) & \\
\hline & gynecology & $104(26.2)$ & \\
\hline & head \& neck & $68(17.1)$ & \\
\hline & urology & $57(14.4)$ & \\
\hline & orthopedic & $35(8.8)$ & \\
\hline & others & $16(4.0)$ & \\
\hline \multicolumn{4}{|c|}{ Anesthetic technique } \\
\hline & general & $232(58.4)$ & \\
\hline & regional & $139(35.0)$ & \\
\hline & combined & $26(6.6)$ & \\
\hline \multirow[t]{4}{*}{ Visit time (hr) } & & & $1.3 \pm 0.6$ \\
\hline & 1 & $270(68.0)$ & \\
\hline & $3-\mathrm{Feb}$ & $108(27.2)$ & \\
\hline & $>3$ & $19(47.8)$ & \\
\hline
\end{tabular}
hours, $123(30.9 \%)$; and the number of visits of $1.3 \pm 0.6$ times where 1 visit was accounted for by $270(68.0 \%), 2-3$ visits, $108(27.2 \%)$ and $>3$ times, 19 (47.8\%) (Table 2).

Table 2 Demographic characteristics of participants completing Si-2AQ (N=397) 
The 95\% Confidence Interval of completely responsive scores to $\mathrm{Si}-2 \mathrm{AQ}$ in six dimensions was determined by its lower and upper bound (Table 3). A binary logistic regression revealed the predicted dependent variables as Process of Care $(4.39 \pm 0.51)$ and Physical Support $(4.35 \pm 0.66)$ in comparison with Accessibility and Convenience (4.33 \pm 0.56$)$, Communication and Information

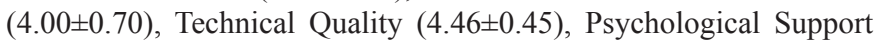
$(4.57 \pm 0.65)$ and Loyalty $(4.52 \pm 0.68)$ in anesthesia quality assessment (Table 4).

Table 3 The $95 \%$ Confidence interval of completely responsive scores to Si-2AQ in 7 dimensions

\begin{tabular}{|c|c|c|c|}
\hline \multirow[t]{2}{*}{ Dimensions } & \multirow[t]{2}{*}{ Responsive score (mean \pm SD) } & \multicolumn{2}{|c|}{ 95\% Confidence Interval } \\
\hline & & Lower & Upper \\
\hline Accessibility & $4.33 \pm 0.56$ & 4.28 & 4.38 \\
\hline Communication & $4.00 \pm 0.70$ & 3.95 & 4.07 \\
\hline Process of care & $4.39 \pm 0.51$ & 4.33 & 4.43 \\
\hline Technical quality & $4.46 \pm 0.45$ & 4.42 & 4.51 \\
\hline Psychological support & $4.57 \pm 0.65$ & 4.51 & 4.62 \\
\hline Physical support & $4.35 \pm 0.66$ & 4.29 & 4.41 \\
\hline Loyalty & $4.52 \pm 0.68$ & 4.47 & 4.59 \\
\hline
\end{tabular}

Table 4 A stepwise binary logistic regression revealed the predicted variables as process of care (PC) and physical support (PHY)

\begin{tabular}{llllll}
\hline & & Sig. & OR & \multicolumn{2}{c}{ 95\% Confidence Interval for CRS } \\
\hline & & & Lower & Upper \\
\hline \multirow{2}{*}{ Step 2 ${ }^{b}$} & PC_mean & 0.006 & 3.139 & 1.393 & 7.074 \\
& PHY_mean & 0.017 & 2.457 & 1.176 & 5.136 \\
\hline
\end{tabular}

$* p<0.05$ statistical significance

\section{Discussion}

The 40-item Si-2-AQ was set and validated for its appropriateness of assessing patients' perspectives on anesthesia care. There were 397 qualified patients, aged $47.6 \pm 16.6$ years, representing all anesthesia services and procedures, who accomplished the questionnaires. Process of Care and Physical Support were found to be significantly valued features in comparison with Accessibility and Convenience, Communication and Information, Technical Quality and Psychological Support in anesthesia quality assessment.

Actually, Process of Care is a core means in anesthesia care service; to elaborate, patients are enabled to feel what would actually happen in the perioperative field. ${ }^{8}$ If the communication period between anesthetists and patients is done well, this might lead to complete understanding and response to anesthesia care. Alghamdi confirmed that health service quality, particularly the empathy and tangible dimension, influenced patient satisfaction. ${ }^{9}$ However, it is questionable whether this was genuinely postoperative patients' reaction, since they were probably too tired to acknowledge a hasty surrounding environment. This seemed to agree with Jenkinson and colleagues' study on patients' experiences and satisfaction with health care: results of a questionnaire study of specific aspects of care. They claimed that patient satisfaction scores represented a limited and optimistic picture. Detailed questions about specific aspects of patients' experiences were likely to be more useful for monitoring the performance of various medical units and could point to how the delivery of health care could be improved. ${ }^{10}$

In addition, warmth and tender health care in the preanesthesia care unit, cleanliness and quiet atmosphere in the operating theatre are the attractive to the public. ${ }^{11}$ Entering a plain tidy room amidst the calm atmosphere, patients would feel serene and tranquil. Eventually, their anxious and nervous attitudes are easily subsided by simple Physical Support. They then feel safe and secure with a willingness to collaborate on intraoperative and postoperative management. Many studies reported that Communication and Information was the main problems in anesthesia quality assessment. ${ }^{12}$ The interaction between caregivers and their customers becomes weak and worse through miscommunication. As a rule, patients do not really know their anesthetists. They are mere acquaintances. ${ }^{13,14}$ Their roles in anesthesia hardly suit patients' culture and background and can limit objective assessment on the quality of anesthesia care.

Though anesthesia service is the medical specialty that focuses on perioperative medicine and the administration of anesthetics, traditionally as the conditions of unconsciousness or sedation with no pain perception during surgical procedure. ${ }^{15}$ Ironically, very few people know what it is all about. This might due to anesthetists and their responsibilities being dominated and concealed by surgical matters. For example, most patients focus their concerns on surgical wound pain. If they have much discomfort perioperatively, anesthesia 
staff are notified and blamed for that. But when patients experience painless conditions, it is often to the surgeons that they first feel grateful. Apparently, patients cannot discriminate between the two authorities on these very close businesses. They do not recognize it as anesthetists' expertise, but as the expertise of surgical operators and their team. ${ }^{16}$ Thus, anesthesia is a thankless job. To improve the situation, anesthetists must introduce themselves to the public, spend more available time to explain details of anesthesia procedures, listen to patients' uneasiness, and learn how to serve and please their customers the best way they can.

Accessibility and Convenience are still the problems faced by all tertiary, university hospitals where demand exceeds supply. ${ }^{17,18}$ Still, long-standing institutes with quite a few physicians in specific fields have become nationally renowned. Thus, patients particularly in a serious situation may start out with very high expectation of the standard of care. ${ }^{19}$ Yet, vulnerable patients such as the low-income or poorly educated group have very few choices for medical treatment; a university hospital could be their last shelter. Regarding patients' perspectives in this dimension in anesthesia care, not only the queueing system, a priority lane or a fast track for patients undergoing surgical procedures, but also respectful anesthesia connection at mutually convenient time is an effective means to relax patients' moods and alleviate this matter.

Generally, perioperative safety and security are the main concerns among patients undergoing a state of unconsciousness. The feeling of vulnerable and scare of painful surgery multiplies the burden of anxiety. Patients are in much greater need for Psychological Support than their physical problems. ${ }^{20}$ Therefore, during the preoperative visit, anesthesia personnel should, as much as possible, leverage this period to introduce themselves to patients, build interpersonal rapport and deliver information about continuous anesthesia service. According to the Protection of Consumers' Rights, having medical personnel propose the choice of anesthesia and procedural details upon patients' preferences is a good way to show respect to each other. ${ }^{21}$

\section{Limitations of the study}

The current study revealed that patients' reactions featured a positive skew in all dimensions. As a matter of fact, we doubted that this reflected genuine responses, since it is undeniable that the wellvalidated $\mathrm{Si}-2-\mathrm{AQ}$ and other psychometric tools still have unavoidable contamination with some halo effects including consideration, doctor-patient relationship, loyalty and faith in the institute, patients' low-income, level of education and past experience in service. The consequences could distort factual relationship and the importance of patient satisfaction. It must also be emphasized that we recorded only patients undergoing anesthesia care in a single university hospital.

\section{Future studies}

We recommend that future studies should develop and validate a "free-halo effects" tool to assess patient satisfaction. The idea proposed by Bauer and co-workers, who suggested the use of a standardized interview instead of questionnaire is worth consideration. ${ }^{22}$ The necessity of Communication and Information should be emphasized; equally important, one should assess patients after their full recovery from unconsciousness.

\section{Conclusion}

The suitability of a developed, well-validated 40-item Si-2-AQ was applied to 397 patients' perspectives on anesthesia care service.
Process of Care and Physical Support appeared to be the main factors, whereas Communication and information, Accessibility and convenience, Technical quality and Psychological support were weak points in anesthesia quality assessment.

\section{Acknowledgments}

The authors thank Mr. Konthi Kulachol for language editing and proofreading of the manuscript.

\section{Funding}

This study is supported by Siriraj Research Development Fund (Managed by Routine to Research: R2R 401/17).

\section{Conflicts of interests}

The authors declare no conflict of interests.

\section{Reference}

1. Faculty of medicine Siriraj Hospital Mahidol University. Annual report 2016.

2. Saul McLeod. Simply psychology. Maslow's Hierarchy of Needs. 2018.

3. Barnett SF, Alagar RK, Grocott MPW, et al. Patient-satisfaction measures in anesthesia: Qualitative systematic review. Anesthesiology. 2013;119(2):452-478.

4. Kutney-Lee A, McHugh MD, Sloane DM, et al. Nursing: A key to patient satisfaction. Health Aff (Millwood). 2009;28(4):w669-77.

5. Holikatti PC, Kar N, Mishra A, et al. A study on patient satisfaction with psychiatric services. Indian J Psychiatry. 2012;54(4):327-332.

6. Brendan EA. Use of ServQUAL in the Evaluation of Service Quality of Academic Libraries in Developing Countries. (2014).

7. Myles PS, Weitkamp K, Melick J, et al. Validity and reliability of a postoperative quality of recovery score: the QoR40. Br J Anaesth. 2000;84:11-15.

8. Gillis C, Gill M, Marlett N, et al. Patients as partners in enhanced recovery after surgery: a qualitative patient-led study. BMJ Open. 2017;7(6):e017002.

9. Alghamdi FS. The impact of service quality perception on patient satisfaction in Government Hospitals in Southern Saudi Arabia. Saudi Med J. 2014;35(10):1271-1273.

10. Jenkinson C, Coulter A, Bruster S, et al. Patients' experiences and satisfaction with health care: results of a questionnaire study of specific aspects of care. Qual Saf Health Care. 2002;11(4):335-339.

11. Gebremedhn EG, Chekol WB, Amberbir WD, et al. Patient satisfaction with anesthesia services and associated factors at the University of Gondar Hospital, 2013: a cross-sectional study. BMC Res Notes. 2015;8:377.

12. Heidegger T, Husemann Y, Nuebling M, et al. Patient satisfaction with anesthesia care: development of a psychometric questionnaire and benchmarking among six hospitals in Switzerland and Austria. $\mathrm{Br} J$ Anaesth. 2002;89(6):863-872.

13. Mako T, Svanang P, Bjersa K. Patients' perceptions of the meaning of good care in surgical care: a grounded theory study. BMC Nurs. 2016;15:47.

14. Aasa A, Hovback M, Bertero CM. The importance of preoperative information for patient participation in colorectal surgery care. $J$ Clin Nurs. 2012;22(11-12):1604-1612. 
15. Cheng SH, Yang $M$, Chiang T. Patient satisfaction with and recommendation of a hospital: effects of interpersonal and technica aspects of hospital care. Int J Qual Health Care. 2003;15(4):345-355.

16. Rathod Y, Devalkar P, Pawar P, et al. Study of patient satisfaction in anesthesia OPD of tertiary care hospital. J Dent Med Sc. 2015;14:66-76.

17. Farley H, Enguidanos ER, Coletti CM, et al. Patient satisfaction surveys and quality of care: an information paper. Ann Emerg Med. 2014;64(4):351-357.

18. Tsai TC, Orav EJ, Jha AK. Patient Satisfaction and Quality of Surgical Care in US Hospitals. Ann Surg. 2015;261(1):2-8.

19. Whitehead D. Beyond the metaphysical: health-promoting existential mechanisms and their impact on the health status of clients. $J$ Clin Nurs. 2003;12(5):678-688.
20. Zimmers S, Brédart A, Dolbeault S. Psychological support for women after breast cancer.

21. Heidegger T, Nuebling M, Germann R, et al. Patient satisfaction with anesthesia care: information alone does not lead to improvement. Can J Anaesth. 2004;51(8):801-805.

22. Baure $\mathrm{M}$, Bohrer $\mathrm{H}$, Aichele $\mathrm{G}$, et al. Measuring patient satisfaction with anesthesia: perioperative questionnaire versus standardised faceto-face interview. Department of Anesthesia, University of Heidelberg, Germany. Acta Anaesthesiol Scand. 2001;45(1):65-72. 